\title{
Effects of Acute Smoked Marijuana on Complex Cognitive Performance
}

\author{
Carl L. Hart, Ph.D., Wilfred van Gorp, Ph.D., Margaret Haney, Ph.D., Richard W. Foltin, Ph.D.,
} and Marian W. Fischman, Ph.D.

\begin{abstract}
Although the ability to perform complex cognitive operations is assumed to be impaired following acute marijuana smoking, complex cognitive performance after acute marijuana use has not been adequately assessed under experimental conditions. In the present study, we used a within-participant double-blind design to evaluate the effects acute marijuana smoking on complex cognitive performance in experienced marijuana smokers. Eighteen healthy research volunteers ( 8 females, 10 males), averaging 24 marijuana cigarettes per week, completed this threesession outpatient study; sessions were separated by at least 72-hrs. During sessions, participants completed baseline computerized cognitive tasks, smoked a single marijuana cigarette $\left(0 \%, 1.8 \%\right.$, or $\left.3.9 \% \Delta^{9}-\mathrm{THC} w / w\right)$, and completed additional cognitive tasks. Blood pressure, heart rate, and subjective effects were also assessed throughout sessions.
\end{abstract}

Marijuana cigarettes were administered in a double-blind fashion and the sequence of $\Delta^{9}$-THC concentration order was balanced across participants. Although marijuana significantly increased the number of premature responses and the time participants required to complete several tasks, it had no effect on accuracy on measures of cognitive flexibility, mental calculation, and reasoning. Additionally, heart rate and several subjective-effect ratings (e.g., "Good Drug Effect," "High," "Mellow") were significantly increased in a $\Delta^{9}$-THC concentration-dependent manner. These data demonstrate that acute marijuana smoking produced minimal effects on complex cognitive task performance in experienced marijuana users.

[Neuropsychopharmacology 25:757-765, 2001] (C) 2001 American College of Neuropsychopharmacology. Published by Elsevier Science Inc.
KEY WORDS: MicroCog; Marijuana; Cannabis; THC, Cognition; Human; Performance

Marijuana is the most widely used illicit drug in the U.S. and, according to the National Household Survey, its use among young adults has steadily increased over the past three years (Substance Abuse and Mental Health Services Administration 2000). While many us-

From the Division on Substance Abuse, New York State Psychiatric Institute and Department of Psychiatry, College of Physicians and Surgeons of Columbia University, New York, NY (CLH, MH, RWF, MWF); and Department of Psychiatry, Weill Medical College of Cornell University, (WVG) New York, NY.

Address correspondence to: Carl L. Hart, Ph.D., New York State Psychiatric Institute, 1051 Riverside Dr., Unit 120, New York, NY 10032.

Received December 18, 2000; revised March 29, 2001; accepted April 18, 2001.

Online publication: 4/19/01 at www.acnp.org/citations/Npp 041901107. ers perceive marijuana as a relatively innocuous substance, concern has been raised recently regarding possible adverse effects on cognitive functioning (Block and Ghoneim 1993; Pope and Yurgelun-Todd 1996). Findings from previous laboratory studies examining marijuana's effects on cognition have been mixed, even when performance on simple psychomotor tasks has been assessed. For example, reaction time has been reported to be slower following acute marijuana in some experiments (Borg et al. 1975; Wilson et al. 1994); but in other experiments, reaction time has been shown to be unaffected (Heishman et al. 1997). There exists, however, more of a consensus regarding marijuana's effects on memory when tested using free recall methods. Several investigators have found marijuana-associated impairment in immediate or delayed recall (Melges et al. 1970; Tinklenberg et al. 1970; Abel 1971; Miller and Cornett 1978; Heishman et al. 1989). The relative consis- 
tency of memory disruptions following marijuana has led to speculation about other marijuana-induced cognitive deficits.

One such area that has received much conjecture, but little actual formal experimental attention, is complex cognitive performance (e.g., shifting of mental sets, inhibiting of prepotent responses, abstraction) during acute marijuana intoxication. In general, complex cognitive functioning is assumed to be impaired following acute marijuana administration. For example, impaired judgement and difficulty carrying out complex mental processes are included among DSM-IV's (American Psychiatric Association 1994) diagnostic criteria for marijuana intoxication. Despite this DSM-IV inclusion, there is surprisingly little published experimental evidence to document marijuana's effects on a broad range of complex cognitive functioning in humans. Research has been hampered for at least two reasons.

Clinical evaluations of marijuana users often do not control for recency of marijuana use, and highly structured clinical evaluations, accomplished on one-to-one basis, may have limited relevance to other less-structured situations (e.g., the workplace). Recently, Powell and his colleagues developed a computerized clinical neuropsychological battery (MicroCog: Assessment of Cognitive Functioning ${ }^{\mathrm{TM}}$ ) that addresses the second issue above (Powell et al. 1993). This battery can be completed in a short period of time (30 to $45 \mathrm{~min}$ ) and assesses performance in several cognitive domains including attention, memory, reasoning, abstraction, and mental calculation. Most importantly, it is designed to be accomplished by the individual under the control of a standardized computer program, which eliminates possible bias and guidance effects from a human clinician. This battery also allows for standardization of multiple test administrations and gives precise quantification of reaction times.

The purpose of this outpatient study was to assess the effects of acute marijuana intoxication on cognitive performance in humans under controlled laboratory conditions. Using a within-participant double-blind design, experienced marijuana smokers participated in 3 experimental sessions during which they smoked one marijuana cigarette $\left(0 \%, 1.8 \%\right.$, or $3.9 \% \Delta^{9}$-tetrahydrocannabinol $\left[\Delta^{9}-\mathrm{THC}\right] \mathrm{w} / \mathrm{w}$ ) each session. The computerized MicroCog ${ }^{\mathrm{TM}}$ and other standardized laboratory assessment batteries (Haney et al. 1997) were used to assess cognitive performance.

\section{METHODS}

\section{Participants}

Eighteen healthy research volunteers, ranging in age from 21 to 35 years (mean $=25.1$ ), completed this threesession outpatient study. Of these participants, eight were female (White-Americans) and ten were male (four Black-Americans, three Hispanic-Americans, three White-Americans). Participants' completed formal education ranged from 11 to 16 years $($ mean $=14.1)$. They were solicited via word-of-mouth referral and newspaper advertisement in New York City. Prior to study participation, all participants passed comprehensive medical and psychological evaluations, and were within normal weight ranges according to the 1983 Metropolitan Life Insurance Company height/weight table. Each female volunteer was given a serum pregnancy test during the screening process and a urine pregnancy test before each experimental session. All reported almost daily marijuana use [mean $=6.1$ days $/$ week ( \pm 1.3$)$ ], averaging four cigarettes per day. On average, participants reported smoking marijuana in this pattern for four years $( \pm 2.8)$. Nine participants reported recent alcohol use (ranging between three and 22 drinks per week) and ten participants reported recent tobacco cigarette use (ranging between two and 10 cigarettes per day). Eight participants reported recent caffeine use (ranging between two and 50 cups per week). Other reported drug use was infrequent (see Table 1). Urine toxicology screens confirmed this, as THC was the only drug metabolite present during the screening process.

Participants were told that the purpose of the study was to evaluate the effects of smoked marijuana on cognitive performance. Each participant signed a consent form approved by the New York State Psychiatric Institute's Institutional Review Board. The consent form described the study and detailed any possible risks. At the end of their third session, participants were fully in-

Table 1. Number of Occasions Participants Reported Ever Having Used Drug of Abuse, Other Than Marijuana

\begin{tabular}{lcccc}
\hline Participant & Cocaine & MDMA & LSD & "Mushrooms" \\
\hline 1 & 0 & 0 & 0 & 0 \\
2 & 4 & 3 & 1 & 1 \\
3 & 0 & 0 & 0 & 0 \\
4 & 2 & 0 & 0 & 0 \\
5 & 2 & 0 & 0 & 0 \\
6 & 1 & 0 & 2 & 0 \\
7 & 1 & 3 & 10 & 0 \\
8 & 2 & 0 & 0 & 0 \\
9 & 1 & 0 & 0 & 0 \\
10 & 10 & 0 & 0 & 0 \\
11 & 0 & 0 & 0 & 0 \\
12 & 0 & 0 & 0 & 0 \\
13 & 0 & 2 & 1 & 2 \\
14 & 0 & 0 & 1 & 1 \\
15 & 0 & 0 & 0 & 0 \\
16 & 4 & 2 & 1 & 1 \\
17 & 0 & 0 & 0 & 0 \\
18 & 0 & 0 & 3 & 3
\end{tabular}

MDMA, methylenedioxymethamphetamine; LSD, lysergic acid diethylamide; Mushrooms, psilocybin. 
formed about experimental and drug conditions, and were paid for their participation.

\section{Procedure and Design}

As a safety precaution, participants agreed that they would not drive to and from the laboratory. All were provided with fare for public transportation. After satisfying all study entry criteria, volunteers received two 3-4 hours-training sessions designed to familiarize them with the daily routine and to provide practice on a standard laboratory assessment battery (Haney et al. 1997); participants did not receive training on the clinical neuropsychological battery. On a different day, participants smoked a sample 3.9\% $\Delta^{9}$-THC concentration marijuana cigarette. The purpose of administering marijuana before the study began was to provide volunteers with experience with the highest $\Delta^{9}$-THC concentration cigarette prior to study onset and to monitor any potential unusual reaction to the cigarette.

Participants completed three experimental sessions, separated from each other by at least 72-hrs. During sessions, each participant smoked one marijuana cigarette containing one of three $\Delta^{9}$-THC concentrations $\left(0 \%, 1.8 \%\right.$, or $\left.3.9 \% \Delta^{9}-\mathrm{THC} \mathrm{w} / \mathrm{w}\right)$. Marijuana cigarettes were administered in a double-blind fashion and the sequence of THC concentration order was balanced across participants using a latin square design.

\section{Experimental Session}

For experimental sessions, participants reported to the laboratory either at 0900 or $1200 \mathrm{hrs}$ and remained for approximately four hours. The session start time for all 3 sessions was constant for each participant. Volunteers were instructed to refrain from using any psychoactive drugs, with the exception of alcohol, caffeine, marijuana, and nicotine. To assure compliance with this requirement, at the start of each study session, volunteers submitted a urine sample that was tested for several drug metabolites (amphetamines, cocaine, and morphine derivatives). Additionally, participants provided a breath sample for the detection of recent alcohol use, and were required to pass a field sobriety test. No urine sample was positive for any drug metabolite, other than THC, and no breath alcohol concentrations were detected for any participant. During sessions, participants were not permitted to smoke tobacco cigarettes.

Following study enrollment, volunteers selected a light meal (breakfast or lunch) and this meal was served to them before each experimental session. Individuals who regularly consumed caffeine had the option to include with their meal a caffeinated beverage. Following meal consumption, participants were seated in front of a Macintosh computer (Cupertino, CA) and monitor with a mouse manipulandum. Then, baseline measures were assessed: 1) heart rate and blood pressure (Sentry II, Model 6100 automated vital sign monitor; NBS Medical, Costa Mesa, CA); and 2) completion of a standard laboratory assessment battery (described below). Immediately following baseline measures, each participant smoked one marijuana cigarette. Subsequently, heart rate, blood pressure, and assessment battery measures were repeatedly obtained at predetermined timepoints. Additionally, the MicroCog ${ }^{\mathrm{TM}}$ (a neuropsychological assessment battery described below) was administered 20-min after marijuana smoking and was completed only once during each session.

Upon completion of a session, participants were evaluated for signs of intoxication and had to pass a field sobriety test. After satisfying the sobriety requirement, they were provided with fare for public transportation.

\section{Assessment Batteries}

MicroCog ${ }^{T M}$ : Assessment of Cognitive Functioning. This 30- to 45-min computerized battery assessed cognitive functioning in several domains, including reaction time, attention, memory, visuospatial processing, reasoning, flexibility and mental calculation (Powell et al. 1993).

REAction Time. During the reaction time task, participants were instructed to respond (pressing the Enter key on the keyboard) as rapidly as possible to a stimulus. The first five items were an auditory stimulus, which were followed by five visual stimulus items. The final five items required the participant to respond to a visual stimulus that was preceded by an auditory "warning" stimulus. Each stimulus was presented at a variable inter-stimulus rate of 1-5 seconds.

AtTENTION. 1) During the alphabet task, a total of 182 letters were presented randomly, one at a time on the computer screen. Participants were told to select (by pressing the Enter key on the keyboard) letters in alphabetical order. The interval between correct responses varied from two to 20 letters. 2) Attention was also assessed using wordlists, consisting of sequential presentation of 16 words that contained groups of category-related items. Participants were instructed to select all words that were members of a given category (e.g., clothing, occupation). Subsequently, a list of 36 words including the 16 words from above, was presented and the participant had to indicate which words were previously shown.

MEMORY. 1) Immediate memory was assessed using a numbers forward task and a numbers reversed task; participants were instructed to immediately reproduce, in the correct sequence, serially-presented digits in the forward direction and in the reverse direction. 2) Verbal memory was assessed using five subtests. Two brief stories were presented one at a time. Immediately 
following each story, participants were required to complete six multiple-choice recognition questions of several items from the stories. Additionally, 20 to 30 min later participants completed twelve multiplechoice questions concerning each story's content. Finally, a name and address were presented on the screen for participants to memorize for later recognition $(\sim 20-$ min later).

Visuospatial Processing. Two different tasks assessed visuospatial processing. 1) A $3 \times 3$ block matrix was presented in which three to five squares were briefly illuminated (the tic-tac task). Immediately after the presentation, participants were instructed to indicate, using the numbered keypad, which blocks contained the illuminated squares. 2) Clock faces with the hands indicating the time, but without associated hour markings, were presented. Digital choices were offered from which participants had to choose the correct time.

Reasoning and Flexibility. 1) An analogy task was used to assess reasoning skills and an object-match task was used to assess cognitive flexibility skills. During the analogy task, participants were presented with a 2-word set (e.g., Green:Lawn) and a single word (e.g., Black) followed by a blank. Then they were required to select from a list of three choices (e.g., Blue, Coal, Grass) a word that best expressed a similar relationship with the single word followed by a blank (e.g., Black) as the one expressed by the 2-word set (e.g., Green:Lawn). In this example, the word Coal correctly completes the analogy. 2) The object-match task assesses concept formation and cognitive flexibility (i.e., the ability to break mental set). During the object-match task, each participant was presented with four figures on the screen. In trial 1, participants were required to identify the one figure that did not match the other three figures. Then, in trial 2, participants were asked to group the figures according to a different principle. Thus, the objectmatch task required participants to identify a concept that was relevant for three of the figures and then shift to another concept that was applicable to three others.

Mental Calculation. The math task measured the ability to perform basic arithmetic operations and was designed to test participants' ability to do mental computations. During the math task, participants completed addition, subtraction, multiplication, and division problems, which varied in difficulty.

Standard Laboratory Battery. The standard laboratory computerized assessment battery completed by participants was composed of a subjective-effects questionnaire and performance task battery. Subjective responses were assessed using a 50-item visual analog scale (VAS), which consisted of a series of lines, each $10 \mathrm{~cm}$ long and labeled "not at all" at one end and "extremely" at the other end (Folstein and Luria 1973). These lines are labeled with adjectives describing a mood (e.g., "I feel . . .
"Anxious," "Angry," "Frustrated"), a drug effect (e.g., "I feel ... "High," "Good Drug Effect," "Bad Drug Effect"), or a physical symptom (e.g., "I feel . . . "Headache," "Stomach Upset," "Muscle Pain"). The VAS was completed at baseline and 7-, 90-, and 140-min post marijuana administration; participants were instructed to complete the subjective-effects questionnaire according to how they felt at the time.

The performance measures included a digit recall task, digit-symbol substitution task (DSST), divided attention task (DAT), and repeated acquisition task (RAT).

Digit ReCALL TASK. During this task, an 8-digit number was displayed for $3 \mathrm{sec}$ on the computer screen. Participants were instructed to enter the number correctly while it was on the screen and again after it had disappeared from the screen. They were also told that they would be asked to reproduce and recognize the number near the end of the battery. This task was designed to assess changes in immediate and delayed recall.

DSST. This 3-min task (McLeod et al. 1982) consisted of nine random three-row $\times$ three-column squares (one square blackened/row) displayed across the top of the computer screen. Each array was associated with a number (1-9). A randomly generated number appeared at the bottom of the screen, indicating which of the arrays should be reproduced on the ninekey keypad attached to the computer. Participants were instructed to reproduce as many patterns as possible by entering the patterns associated with the randomly generated numbers.

DAT. This 5-min task consisted of concurrent pursuit-tracking and vigilance tasks (Miller et al. 1988). Participants tracked a moving circle on the video screen using the mouse, and also had to signal when a small black square appeared at any of the four corners of the screen. Accurate tracking of the moving stimulus increased its speed proportionately.

RAT. At the start of this 3-min learning task, four buttons were illuminated, and participants were instructed to learn a 10-response sequence of button presses. A position counter incremented by one each time a correct button was pressed, and remained unchanged after an incorrect response. The points counter increased by one each time the 10-response sequence was correctly completed. The sequence remained the same throughout the task, but a new random sequence was generated when the task occurred again. Participants were instructed to earn as many points during the task as possible by pressing the buttons in the correct sequence.

\section{Drug}

During each session, participants smoked a single onegram marijuana cigarette $\left(0,1.8,3.9 \% \Delta^{9}-\mathrm{THC} \mathrm{w} / \mathrm{w}\right.$, 
provided by the National Institute on Drug Abuse). Participants took three standardized puffs from the marijuana cigarette: each puff consisted of a 5-sec preparation interval, followed by 5 -sec of inhalation, $10-\mathrm{sec}$ of breath-hold, and 40-sec of exhalation and rest. Cigarettes were tightly rolled at both ends and were smoked through a hollow plastic cigarette holder so that the contents were not visible. Twenty-four hours prior to administration, cigarettes were removed from a freezer, where they were stored in an airtight container, and humidified at room temperature.

\section{Data Analysis}

Planned comparisons were used to determine the effects of $\Delta^{9}$-THC concentration $\left(0,1.8,3.9 \% \Delta^{9}\right.$-THC) on MicroCog ${ }^{\mathrm{TM}}$ performance. The following comparisons were performed: 0 vs. $1.8 \%, 0$ vs. $3.9 \%$, and 1.8 vs. $3.9 \%$. A 3 (dose) $\times 4$ (time) repeated measures ANOVA was used to examine differences between THC concentrations on subjective ratings and psychomotor performance and a 3 (dose) $\times 5$ (time) repeated measures ANOVA was used to assess $\Delta^{9}$-THC-concentration effects on heart rate and blood pressure. As described above, three planned comparisons were performed. Hunyh-Feldt corrections were used and only those $p$-values $<.05$ were considered statistically significant.

\section{RESULTS}

\section{Cognitive Effects}

MicroCog ${ }^{T M}$. Figure 1 (upper panel) shows the mean number of premature responses on the reaction time task as a function of $\Delta^{9}$-THC concentration. Premature responses significantly increased following administration of cigarettes containing 3.9\% $\Delta^{9}$-THC concentration, compared to when participants smoked $0 \%$ and $1.8 \% \Delta^{9}$-THC concentration cigarettes $(p<.05)$.

The effects of marijuana on time required to complete selected cognitive tasks are displayed in the lower panel of Figure 1. The amount of time participants needed to read the brief stories (for later memorization), to complete the immediate recall, tic-tac, clocks (data not shown) and mental calculation tasks significantly increased under the $3.9 \% \Delta^{9}$-THC concentration condition, relative to the $0 \% \Delta^{9}$-THC condition $(p<$ $0.05)$. Also, when compared to the $1.8 \% \Delta^{9}$-THC condition, $3.9 \% \Delta^{9}$-THC concentration cigarettes significantly increased the amount of time it took participants to finish the immediate recall and mental calculation tasks ( $p<$ .05). No significant differences were observed when accuracy of responding on attention, memory, visuospatial processing, reasoning, flexibility, and mental calculation tasks were analyzed as a function of $\Delta^{9}$-THC concentration.
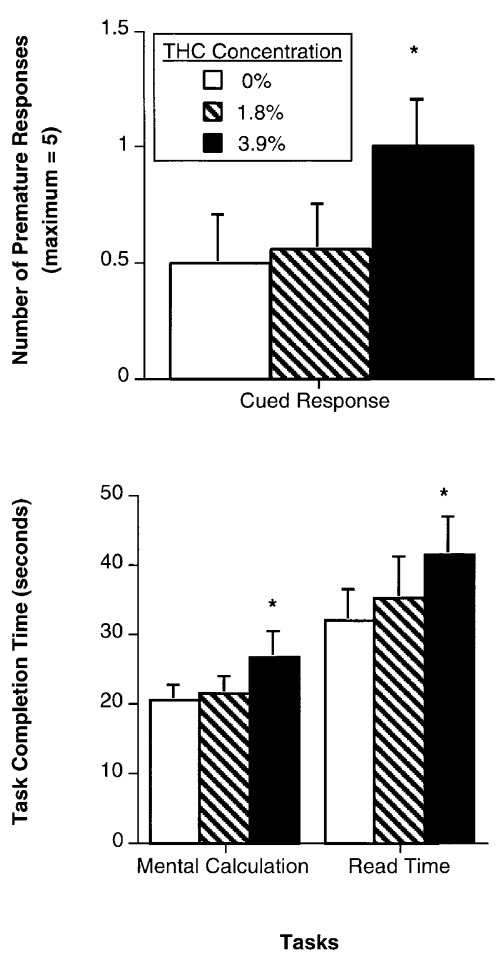

Figure 1. Upper Panel: Mean total number of premature responses as a function of $\Delta^{9}$-THC concentration. Under each dosing condition, participants had five trials. Lower Panel: Mean amount of time participants required to complete the mental calculation task (left) and mean amount of time participants required to read and memorize short stories (right). Error bars represent one SEM. An * indicates that the $3.9 \% \Delta^{9}$-THC condition significantly differed from the other two conditions $(p<.05)$.

Standard Laboratory Battery. Performance on the digit immediate recall task was impaired following administration of $3.9 \% \Delta^{9}$-THC cigarettes: the percentage of digits correctly entered was significantly decreased compared to when $0 \%$ and $1.8 \% \Delta^{9}$-THC cigarettes were administered. Performance on the tracking portion of the DAT was improved following the high $\Delta^{9}$-THC concentration cigarettes relative to the other conditions $(p<.05$; data not shown). No other significant performance effects were noted.

\section{Subjective Effects}

Figure 2 illustrates the effect of smoked marijuana on selected subjective ratings as a function of $\Delta^{9}$-THC concentration and time. Ratings were significantly elevated in a $\Delta^{9}$-THC concentration-dependent fashion: participants' ratings of "Bad Drug Effect," "Can't Concentrate," "Confused," "Forgetful," "Mellow," (data not shown) "Good Drug Effect," and "High" were significantly increased after participants smoked cigarettes containing $3.9 \% \Delta^{9}$-THC compared to when they smoked $0 \%$ and $1.8 \% \Delta^{9}$-THC cigarettes $(p<.05)$ (see also 


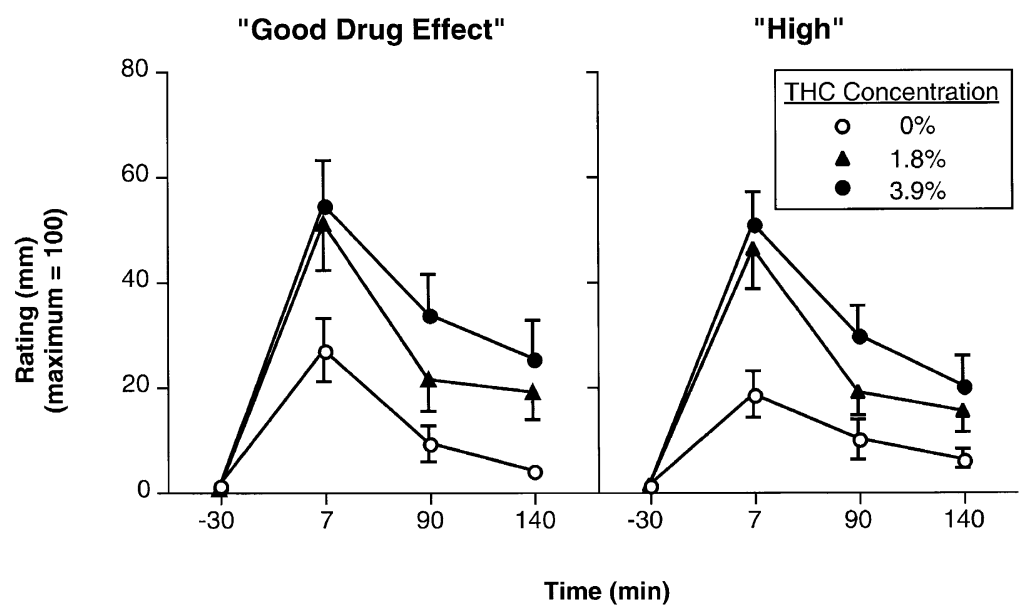

Figure 2. Mean subjective-effects ratings as a function of $\Delta^{9}$-THC concentration and time. Error bars represent one SEM. See text for details.
Table 2). Relative to $0 \% \Delta^{9}$-THC cigarettes, $1.8 \% \Delta^{9}$-THC cigarettes produced significant effects on ratings of "Good Drug Effect," "Heart Pounding," "High," "Hungry," "Sedated," and "Stimulated" $(p<.05$; data shown only for "Good Drug Effect and "High").

\section{Physiological Effects}

Figure 3 presents effects of smoked marijuana on heart rate as a function of $\Delta^{9}$-THC concentration and time. The repeated-measures ANOVA revealed a significant $\Delta^{9}$-THC concentration $\times$ time effect $[F(8,136)=13.31, p<$ .05]. Relative to placebo $\Delta^{9}$-THC condition, both $1.8 \%$ and $3.9 \% \Delta^{9}$-THC significantly increased bpm $(p<.05)$. Additionally, $3.9 \% \quad \Delta^{9}$-THC produced significantly higher heart rates than did $1.8 \% \Delta^{9}$-THC $(p<.05)$. No significant effects of smoked marijuana on blood pressure were observed.

\section{DISCUSSION}

Data from the present investigation clearly show that smoked marijuana produced $\Delta^{9}$-THC concentrationdependent increases in a number of subjective-effect ratings and heart rate, while producing minimal effects on cognitive performance. Marijuana increased premature responding and the amount of time needed to complete several MicroCog ${ }^{\mathrm{TM}}$ tasks, including the immediate recall, tic-tac, clocks, and mental calculation tasks. Nevertheless, accuracy on these and other cognitive tasks were unaltered after marijuana administration. Consis-

Table 2. Mean Subjective-Effects Ratings after 3.9\% $\Delta^{9}$-THC and after $0 \% \Delta^{9}$-THC

\begin{tabular}{|c|c|c|c|c|c|}
\hline \multirow[b]{2}{*}{ Measure } & \multicolumn{2}{|c|}{ 0\% $\Delta^{9}$-ТHC } & \multicolumn{2}{|c|}{$3.9 \% \Delta^{9}$-THC } & \multirow[b]{2}{*}{ F Value } \\
\hline & Mean & SEM & Mean & SEM & \\
\hline \multicolumn{6}{|c|}{ Scales on which marijuana increased ratings } \\
\hline Bad drug effect & 2.39 & 0.32 & 5.07 & 0.51 & 15.35 \\
\hline Can't concentrate & 7.63 & 2.56 & 14.10 & 2.19 & 9.92 \\
\hline Confused & 2.74 & 0.46 & 4.89 & 0.47 & 4.98 \\
\hline Forgetful & 8.04 & 0.73 & 14.65 & 0.88 & 18.96 \\
\hline Good drug effect & 13.10 & 6.96 & 37.57 & 8.73 & 116.82 \\
\hline Heart pounding & 0.93 & 0.13 & 7.39 & 4.26 & 11.43 \\
\hline High & 11.31 & 3.66 & 33.33 & 9.02 & 91.85 \\
\hline Hungry & 12.10 & 3.98 & 20.89 & 5.65 & 13.40 \\
\hline Jittery & 1.70 & 0.26 & 4.06 & 3.22 & 4.71 \\
\hline Mellow & 29.57 & 1.04 & 41.02 & 3.04 & 21.42 \\
\hline Noise seem louder & 0.96 & 0.07 & 4.19 & 1.19 & 8.84 \\
\hline Sedated & 7.13 & 0.89 & 14.70 & 1.88 & 19.34 \\
\hline Stimulated & 12.56 & 2.81 & 26.44 & 4.88 & 28.20 \\
\hline Withdrawn & 3.06 & 0.53 & 9.09 & 2.05 & 12.89 \\
\hline
\end{tabular}

All $F$ values were significant at $p<.05$. 


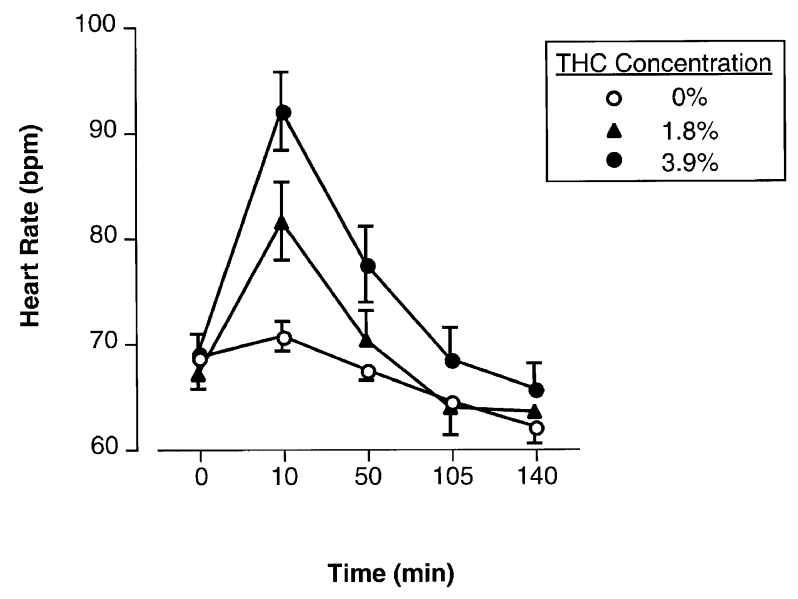

Figure 3. Selected mean heart rate as a function of $\Delta^{9}$-THC concentration and time. Error bars represent one SEM. See text for details.

tent with MicroCog ${ }^{\mathrm{TM}}$ results, data from the standard laboratory battery indicated that psychomotor performance was only subtly affected by smoked marijuana: following high $\Delta^{9}$-THC concentration cigarettes, participants exhibited a decrement in performance only during the immediate recall task, while performance on a tracking task was markedly improved.

The most salient cognitive effect from the present investigation was the finding that premature responses were increased after volunteers smoked the high $\Delta^{9}$-THC concentration cigarette. Participants averaged one premature response compared to less than one following smoking the placebo and low $\Delta^{9}$-THC concentration cigarette. Hooker and Jones (1987) previously reported that inhibitory control, as measured by the Stroop test, was impaired during marijuana intoxication in light users. The current results extend these findings by demonstrating that, following acute marijuana smoking, heavy marijuana users also exhibit difficulties inhibiting inappropriate responding. Although marijuana's effects on inhibitory control observed in the present study were not overwhelming, in light of increasingly popular theories emphasizing the importance of inhibition as a basic unit of working memory and executive control processes (Dempster and Corkill 1999; Zacks and Hasher 1994), these results are suggestive of potential executive cognitive functioning problems following acute marijuana use.

Despite the fact that marijuana $\left(3.9 \% \Delta^{9}\right.$-THC) increased the amount of time participants required to complete several tasks, no impairments in accuracy were observed during MicroCog ${ }^{\mathrm{TM}}$ tasks and the only performance decrement observed during the standard laboratory battery was the percentage of correct digits recalled. The relatively few accuracy impairments observed is congruent with several other studies investigating acute marijuana effects on psychomotor and simple cognitive performance (Chait and Perry 1994; Azorlosa et al. 1995; Fant et al. 1998). Moreover, the present data expands these findings by showing that more complex cognitive performance is only minimally affected following acute marijuana smoking.

It should be noted, however, that some studies have reported data showing diminished performance on the DAT (Casswell and Marks 1973; Barnett et al. 1985; Perez-Reyes et al. 1988; Marks and MacAvoy 1989), the DSST (Heishman et al. 1989, 1997; Kelly et al. 1993: Wilson et al. 1994; Haney et al. 1997, 1999), and the RAT (Heishman et al. 1989; Foltin et al. 1993; Kelly et al. 1993; Haney et al. 1997) following similarly tested concentrations of $\Delta^{9}$-THC. One possible reason we did not observe any performance decrement on these tasks is because the MicroCog ${ }^{\mathrm{TM}}$, which required 30-45 min for completion, was administered during peak marijuana intoxication and participants' post-marijuana performance on the DAT, DSST, and RAT occurred under less than peak marijuana-related subjective and physiological effects. This explanation seems unlikely because some of the tasks from MicroCog ${ }^{\mathrm{TM}}$ assessed similar domains as those measured by the DAT, DSST, and RAT. Heishman et al. (1997) also found that performance on word free recall tasks was impaired following marijuana ( $3.55 \% \Delta^{9}$-THC).

It is possible that the cognitive tests used in the current study were insensitive to marijuana's effects. For instance, most of the memory tests from MicroCog used a multiple-choice format, placing less demand on participants' memory than would a free recall task. This speculation does not adequately explain our current findings because the numbers forward and numbers reverse tasks were not multiple-choice and relied on attention and working memory. Participants' accuracy on these tasks exhibited little or no change across experimental sessions, consistent with accuracy of performance on other memory tests. A more parsimonious explanation is that the participants tested in the current study were tolerant to many marijuana-related cognitive effects, as they reported substantially greater current marijuana use than did volunteers in most other studies reporting performance decrements after smoked marijuana. On average, participants smoked six days per week, averaging four marijuana cigarettes per day. In support of marijuana tolerance, several other investigators have reported data demonstrating that, relative to light marijuana smokers, heavy marijuana smokers show less cognitive impairment following acute marijuana administration (Meyer et al. 1971; Rickles et al. 1973; Cohen and Rickles 1974).

While heavy marijuana use may account for differences between the current cognitive data and past findings, heavy marijuana use alone does not result in diminution of all marijuana-related effects. For example, in the present study, marijuana produced $\Delta^{9}$-THC concen- 
tration-dependent increases on several subjective effects (e.g., "Good Drug Effect," "High," "Mellow") in heavy marijuana smokers. Similar findings have been reported when light marijuana users were assessed under comparable laboratory conditions (Kelly et al. 1993; Fant et al. 1998). Moreover, heart rate data from the current investigation showed a pattern analogous to subjective-effect ratings, such that heart rate was increased in a $\Delta^{9}$-THC concentration-dependent manner. In agreement with previously published studies that have examined marijuana-associated subjective effects in frequent and infrequent marijuana users, frequent users' heart rate responses have also been shown to be comparable to those of infrequent users following acute marijuana smoking (Casswell and Marks 1973; Lindgren et al. 1981). These findings suggest that marijuana use history plays less of a role in subjective ratings and heart rate responses than it does on cognitive performance after smoking a single marijuana cigarette.

Behavioral factors could account for the differential development of tolerance to effects produced by smoked marijuana. Regarding cognitive performance, it is possible that regular marijuana users (through repeated practice) have learned to be more cautious when executing challenging activities (e.g., complex cognitive operations) during intoxication. As such, the likelihood of observing performance impairments in this group, relative to less frequent marijuana users, is decreased. But, as increased caution is exercised, in order to ensure precise functioning, more time may be required for task completion. In fact, the results of the current investigation support this hypothesis: the amount of time necessary for participants to complete several cognitive tasks significantly increased after smoking marijuana, whereas task accuracy rates were only slightly affected. In contrast, subjective-effect ratings and heart rate response did not show a corresponding tolerance after volunteers smoked a single marijuana cigarette. Becker (1953) suggested that before individuals are able to experience marijuana-associated positive subjective effects, they must go through a process in which they learn to recognize and interpret psychoactive effects produced by smoked marijuana. As one acquires more experience with smoking marijuana, one becomes more competent in identifying marijuana-related subjective effects, possibly decreasing the prospect of substantial tolerance to marijuana's subjective effects. Additionally, Schuster et al. (1966) hypothesized that tolerance will develop to drug-related behaviors that are disruptive to an individual's ability to meet performance requirements necessary for the delivery of reinforcers. Conversely, tolerance will not develop to drug-related behaviors that facilitate or have no effect on an individual's ability to satisfy necessary operations for reinforcement delivery. Ergo, it is possible that marijuana-related cognitive performance is under contingency control such that im- paired performance may result in a decreased delivery of reinforcers, whereas subjective effects are not under such control. Because heart rate is a relatively stable physiological measure, it is unlikely that behavioral factors are involved in the lack of tolerance observed after smoking a single marijuana cigarette. Indeed, heart rate increases after smoked marijuana may serve as a conditioned cue to experienced marijuana smokers, which aid in identifying some of marijuana's imminent psychoactive effects.

In summary, while subjective ratings and heart rate were significantly elevated in a $\Delta^{9}$-THC concentrationrelated fashion, cognitive performance was minimally affected following acute marijuana smoking. Participants experienced greater difficulties inhibiting inappropriate responding following the high $\Delta^{9}$-THC concentration cigarette and they required more time to complete several cognitive tests, but their test accuracy rates were unaffected. The finding that accuracy was unaffected by smoked marijuana indicates that heavy, daily, marijuana smokers will not fulfill the DSM-IV criterion for marijuana intoxication that requires impairment of complex cognitive functioning. The consistent slowing of cognitive performance may have significant behavioral effects under some circumstances requiring complex operations that must be accomplished in a limited time frame, such as certain workplace tasks and the operation of machinery and automobiles.

The current finding suggest that it would be useful to include slowing of cognitive performance as a component of the assessment of cognitive impairment when applying the DSM-IV criteria for marijuana intoxication. Furthermore, these data underscore the importance of taking into account the marijuana use history of research participants when assessing the impact of marijuana smoking on cognitive performance.

\section{ACKNOWLEDGMENTS}

This research was supported by DA-03746. The assistance of Shannon Lewis, Rachelle Reis-Larson, Mabel Torres, and Drs. Amie S. Ward, Sandra D. Comer, Evaristo Akerele, and Jeffrey Wilson are gratefully acknowledged.

\section{REFERENCES}

Abel EL (1971): Marihuana and memory: Acquisition or retrieval? Science 173:1038-1040

American Psychiatric Association (1994): Diagnostic and Statistical Manual of Mental Disorders, 4th ed. Washington D.C., American Psychiatric Association

Azorlosa JL, Greenwald MK, Stitzer ML (1995): Marijuana smoking: Effects of varying puff volume and breathhold duration. J Pharmacol Exp Ther 272:560-569 
Barnett G, Licko V, Thompson T (1985): Behavioral pharmacokinetics of marijuana. Psychopharmacology 85:51-56

Becker HS (1953): Becoming a marihuana user. Am J Sociol 59:235-242

Block RI, Ghoneim MM (1993): Effects of chronic marijuana use on human cognition. Psychopharmacology 110:219-228

Borg J, Gershon S, Alpert M (1975): Dose effects of smoked marihuana on human cognitive and motor functions. Psychopharmacologia (Berlin) 42:211-218

Casswell S, Marks DF (1973): Cannabis and temporal disintegration in experienced and naïve subjects. Science 179:803-805

Chait LD, Perry JL (1994): Acute and residual effects of alcohol and marijuana, alone and in combination, on mood and performance. Psychopharmacology (Berlin) 115:340-349

Cohen MJ, Rickles WH Jr (1974): Performance on a verbal learning task by subjects of heavy past marijuana usage. Psychopharmacologia 37:323-330

Dempster FN, Corkill AJ (1999): Individual differences in susceptibility to interference and general cognitive ability. Acta Psychologica 101:395-416

Fant RV, Heishman SJ, Bunker EB, Pickworth WB (1998): Acute and residual effects of marijuana in humans. Pharmacol Biochem Behav 60:777-784

Folstein MF, Luria R (1973): Reliability, validity, and clinical application of the visual analogue mood scale. Psychol Med 3:479-486

Foltin RW, Fischman MW, Pippen PA, Kelly TH (1993): Behavioral effects of cocaine alone and in combination with ethanol or marijuana in humans. Drug Alc Depend 32:90-106

Haney M, Comer SD, Ward AS, Foltin RW, Fischman MF (1997): Factors influencing marijuana self-administration by humans. Behav Pharmacol 8:101-112

Haney M, Ward AS, Comer SD, Foltin RW, Fischman MW (1999): Abstinence symptoms following smoked marijuana in humans. Psychopharmacology 141:395-404

Heishman SJ, Stitzer ML, Yingling JE (1989): Effects of tetrahydrocannabinol content on marijuana smoking behavior, subjective reports and performance. Pharmacol Biochem Behav 34:173-179

Heishman SJ, Arasteh K, Stitzer ML (1997): Comparative effects of alcohol and marijuana on mood, memory and performance. Pharmacol Biochem Behav 58:93-101

Hooker WD, Jones RT (1987): Increased susceptibility to memory intrusions and stroop interference effect during acute marijuana intoxication. Psychopharmacology 91:20-24

Kelly TH, Foltin RW, Fischman MW (1993): Effects of smoked marijuana on heart rate, drug ratings and task performance by humans. Behav Pharmacol 4:167-178

Lindgren JE, Ohlsson A, Agurell S, Hollister L, Gillespie H (1981): Clinical effects and plasma levels of ( $\Delta^{9}$-tetrahy- drocannabinol $\left(\Delta^{9}-\mathrm{THC}\right)$ in heavy and light users of cannabis. Psychopharmacology 74:208-212

Marks DF, MacAvoy MG (1989): Divided attention performance in cannabis users and non-users following alcohol and cannabis separately and in combination. Psychopharmacology 99:397-401

McLeod DR, Griffiths RR, Bigelow GE, Yingling J (1982): An automated version of the digit symbol substitution test (DSST). Behav Res Meth Instr 14:463-466

Melges FT, Tinklenberg JR, Hollister LE, Gillespie HK (1970): Marihuana and temporal disintegration. Science 168:1118-1120

Meyer RE, Pillard RC, Shapiro LM, Mirin SM (1971): Administration of marijuana to heavy and casual marijuana users. Am J Psychiatry 128:90-96

Miller LL, Cornett TL (1978): Marijuana: Dose effects on pulse rate, subjective estimates of intoxication, free recall and recognition memory. Pharmacol Biochem Behav 9:573-577

Miller TP, Taylor JL, Tinklenberg JR (1988): A comparison of assessment techniques measuring the effects of methylphenidate, secobarbital, diazepam, and diphenhydramine in abstinent alcoholics. Neuropsychobiology 19:90-96

Perez-Reyes M, Hicks RE, Bumberry J, Jeffcoat AR, Cook CE (1988): Interaction between marijuana and ethanol: Effects on psychomotor performance. Alc Clin Exp Res $12: 268-276$

Pope HG Jr, Yurgelun-Todd D (1996): The residual cognitive effects of heavy marijuana use in college students. JAMA 275:521-527

Powell DH, Kaplan EF, Whitla D, Weintraub S, Catlin R, Funkenstein HH (1993): MicroCog Assessment of Cognitive Functioning. San Antonio, The Psychological Corporation

Rickles WH Jr, Cohen MJ, Whitaker CA, McIntyre KE (1973): Marijuana induced state-dependent verbal learning. Psychopharmacologia 30:349-354

Schuster CR, Dockens WS, Woods JH (1966): Behavioral variables affecting the development of amphetamine tolerance. Psychopharmacologia 9:170-182

Substance Abuse and Mental Health Services Administration (2000): Summary Findings from the 1999 National Household Survey on Drug Abuse. Rockville, Department of Health and Human Services

Tinklenberg JR, Melges FT, Hollister LE, Gillespie HK (1970): Marijuana and immediate memory. Nature 226: 1171-1172

Wilson WH, Ellinwood EH, Mathew RJ, Johnson K (1994): Effects of marijuana on performance of a computerized cognitive-neuromotor test battery. Psychiat Res 51:115-125

Zacks RT, Hasher L (1994): Directed ignoring: Inhibitory regulation of working memory. In Dagenbach D, Carr TJ (eds), Inhibitory Processes in Attention, Memory, and Language. San Diego, CA, Academic Press, pp 241-262 